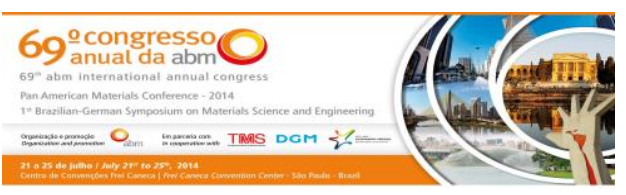

Tema: Corrosão

\title{
INFLUÊNCIA DO ELETRÓLITO NO DESEMPENHO DE FILMES DE POLIPIRROL NA PROTEÇÃO DO ALUMÍNIO 2024 CONTRA A CORROSÃO*
}

\begin{abstract}
Karine Miranda Bezerra' Felipe Ezequiel Pereira dos Santos² Andréa Santos Liu ${ }^{3}$ Liu Yao $\mathrm{Cho}^{4}$
\end{abstract}

\section{Resumo}

A presente pesquisa investigou a eficiência dos filmes de polipirrol (PPy) eletrodepositados potenciostaticamente em meio aquoso contendo os Ácidos: Dodecil Benzeno Sulfônico (DBSA) ou p-Toluenossulfônico (pTS) na proteção da Liga de Alumínio 2024 contra a corrosão. Ensaios de Polarização Potenciodinâmica e Voltametria Cíclica obtidos em $\mathrm{NaCl}$ demonstraram significativa proteção do Alumínio 2024 quando revestido pelos filmes de PPy/pTS, em detrimento do Sistema PPy/DBSA que sofre o processo irreversível de overoxidação. A Morfologia dos filmes avaliada por Microscopia Eletrônica de Varredura (MEV) indicaram que o Sistema PPy/pTS é mais homogêneo e uniforme, quando comparados aos filmes de PPy/DBSA, que apresentaram defeitos estruturais que justificam a menor performance desses filmes na proteção do metal contra a corrosão.

Palavras-chave: Corrosão; Alumínio; Polipirrol; Eletrólito.

\section{INFLUENCE OF ELECTROLYTE ON THE PERFORMANCE OF POLYPYRROLE FILMS TO CORROSION PROTECTION OF 2024 ALUMINUM ALLOY}

\section{Abstract}

In this work was investigated the performance of PPy films electrodeposited in aqueous solution containing aromatic acids - dodecyl benzene sulfonic acid (DBSA) and p-toluene sulfonic acid (pTS) - to protecting aluminum alloy. Potentiodynamic polarization curves and cyclic voltammetry in chloride medium have showed that PPy deposited in pTS presented better performance. This behavior was attributed to higher homogeneity of this polymeric film, which confirmed by Scanning Electronic Microscopy (SEM).

Keywords: Corrosion; Aluminum; Polypyrrole; Electrolyte.

1 Graduanda em Licenciatura em Química, Instituto Federal de Educação, Ciência e Tecnologia de São Paulo (IFSP), São Paulo, Brasil.

2 Mestrando em Processamento de Materiais e Catálise do Instituto de Pesquisa e Desenvolvimento (IP\&D) da Universidade do Vale do Paraíba (UNIVAP), São José dos Campos, São Paulo, Brasil.

3 Doutora em Engenharia Aeronáutica e Mecânica, Docente do Departamento de Química do Instituto Federal de Educação, Ciência e Tecnologia de São Paulo (IFSP), São Paulo, Brasil.

4 Doutor em Química (Ciências), Coordenador do Curso de Graduação em Engenharia Química na Faculdade de Engenharias, Arquitetura e Urbanismo, UNIVAP, São José dos Campos, São Paulo, Brasil.

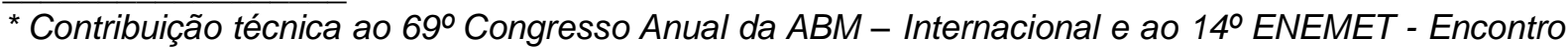
Nacional de Estudantes de Engenharia Metalúrgica, de Materiais e de Minas, 21 a 25 de julho de 2014, São Paulo, SP, Brasil.
} 


\section{INTRODUÇÃO}

O Polipirrol (PPy) é um polímero condutor que têm sido investigado para inúmeras aplicações tecnológicas, destacando-se seu uso em supercapacitores [1-3], nanotubos de carbono [4], baterias recarregáveis [5], membranas [6], sensores [7] e na proteção contra a corrosão [8-10]. Os filmes de PPy podem ser sintetizados por métodos químicos ou eletroquímicos [11], e suas propriedades elétricas, químicas, mecânicas e morfológicas são afetadas por inúmeros fatores experimentais, ressaltando-se a natureza do eletrólito e o potencial aplicado durante a eletrodeposição do filme polimérico [12,13]. A estrutura dos ânions da solução eletrolítica afeta a condutividade, a estabilidade e a morfologia dos filmes de PPy [14], além de influenciar no tipo de óxido de alumínio formado na interface metal/solução.

Filmes poliméricos dopados com ânions orgânicos aromáticos apresentam elevada condutividade, maior homogeneidade e aderência, quando comparados a dopagem com ânions inorgânicos $[15,14]$. Nessa perspectiva, a presente pesquisa retrata a influência de ácidos aromáticos, como os ácidos: dodecil-benzeno sulfônico e ptoluenosulfônico no processo de eletrodeposição de filmes de PPy, bem como a performance desses filmes na proteção da liga de alumínio 2024 contra a corrosão.

\section{MATERIAIS E MÉTODOS}

\subsection{Reagentes, Soluções e Materiais}

A liga de alumínio 2024 é constituída por 4,4\% de cobre, 1,5\% de magnésio e 0,6\% de manganês. Em cada experimento, a superfície metálica foi polida com lixas com granulometria de 360, 600 e 1200. Posteriormente, foi enxaguada com água deionizada. A eletrodeposição dos filmes de PPy foi realizada em meio aquoso contendo 0,2 mol.L-1 de pirrol (Py,) e 0,1 mol.L-1 do eletrólito, ácido dodecil-benzeno sulfônico (DBSA) ou ácido p-toluenossulfônico (pTS). Os testes de polarização potenciodinâmica e voltametria cíclica foram realizados em solução de $\mathrm{NaCl}\left(0,1 \mathrm{~mol}^{-\mathrm{L}^{-1}}\right)$.

Os ensaios eletroquímicos foram realizados em uma célula contendo três eletrodos: (i) eletrodo de trabalho: liga de alumínio 2024 embutida em Teflon ${ }^{\circledR}$ com área exposta de $0,53 \mathrm{~cm}^{2}$; (ii) eletrodo auxiliar: fio de platina e (iii) eletrodo de referência: eletrodo de $\mathrm{Ag} / \mathrm{AgCl}, \mathrm{Cl}^{-}$saturado.

\subsection{Eletrodeposição dos Filmes de PPy}

Os filmes de PPy foram depositados a potencial controlado e à temperatura ambiente, utilizando-se um Potenciostato/Galvanostato modelo MQPG-01 Microquímica, conectado e controlado por um microcomputador. A influência dos ânions foi investigada potenciostaticamente, utilizando-se soluções aquosas de DBSA, pTS e Py. A eletrodeposição dos filmes foi averiguada aplicando-se um potencial de $+1,4 \mathrm{~V} v s$. $\mathrm{Ag} / \mathrm{AgCl}$ durante $900 \mathrm{~s}$.

\subsection{Ensaios de Polarização Potenciodinâmica e Voltametria Cíclica}

Os ensaios de polarização potenciodinâmica e voltametria cíclica foram realizados em solução aquosa de $\mathrm{NaCl} 0,1$ mol.L-1 $(\mathrm{pH} 5,9)$, variando-se o potencial

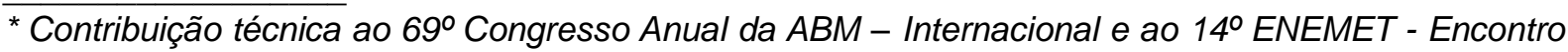
Nacional de Estudantes de Engenharia Metalúrgica, de Materiais e de Minas, 21 a 25 de julho de 2014, São Paulo, SP, Brasil.
} 


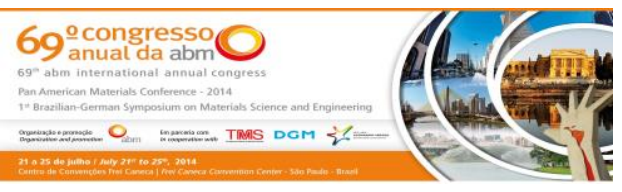

de $-1,5 \mathrm{a}+1,5 \mathrm{~V}$ a uma velocidade de varredura igual a $5 \mathrm{mV} . \mathrm{s}^{-1}$. Os valores de potencial de corrosão ( $E_{\text {corr }}$ ) e densidade de corrente de corrosão (jcorr) foram obtidos a partir das curvas de Tafel; sendo o $\mathrm{E}_{\text {corr }}$ determinado pela intersecção dos ramos anódicos e catódicos quando $\mathrm{j}=0$, e os valores de jcorr extrapolados no potencial de corrosão através da intersecção dos trechos lineares das curvas.

\subsection{Caracterização dos Filmes de PPy}

Os filmes de PPy/DBSA e PPy/pTS foram caracterizados por FTIR, através da preparação de pastilhas de $\mathrm{KBr}$. As condições de análise foram: (1) faixa de comprimento de onda de 4000 a $400 \mathrm{~cm}^{-1}$; (2) resolução de $4 \mathrm{~cm}^{-1}$; (3) 40 scans e (4) temperatura de $25^{\circ} \mathrm{C}$. Os espectros foram obtidos usando-se um espectrômetro modelo SPECTRUM 2000 (Perkin Elmer).

A morfologia das superfícies de alumínio recobertas com PPy foram analisadas utilizando-se um Microscópio Eletrônico de Varredura (MEV) modelo Jeol JXA 840A.

As micrografias foram obtidas utilizando-se um feixe de elétrons de $15 \mathrm{keV}$.

A Figura 1 apresenta um fluxograma das etapas envolvidas no desenvolvimento deste trabalho.

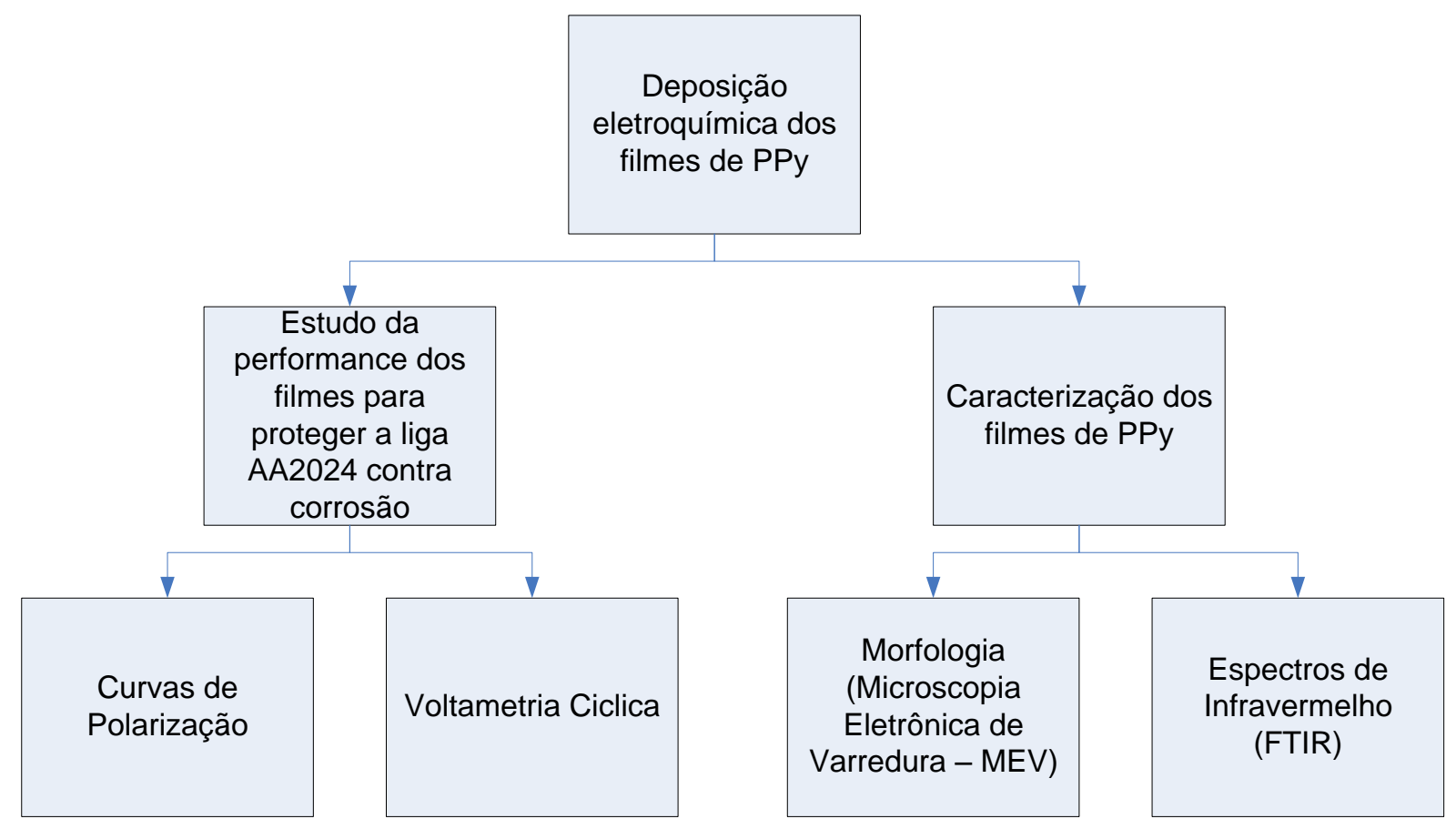

Figura 1. Fluxograma das etapas desenvolvidas no trabalho.

\section{RESULTADOS E DISCUSSÃO}

\subsection{Eletrodeposição dos Filmes de PPy}

A Figura 2 apresenta os voltamogramas cíclicos para a deposição dos filmes de PPy/DBSA (Curva 1) e a influência do DBSA sobre a superfície de alumínio na ausência de Py (Curva 2); variando-se o potencial de $-1,5 \mathrm{~V}$ a $+1,5 \mathrm{~V}$, a velocidade de varredura igual a $5 \mathrm{mV} . \mathrm{s}^{-1}$.

\footnotetext{
* Contribuição técnica ao 69 Congresso Anual da ABM - Internacional e ao 14ํㅡㄹ ENET - Encontro Nacional de Estudantes de Engenharia Metalúrgica, de Materiais e de Minas, 21 a 25 de julho de 2014, São Paulo, SP, Brasil.
} 


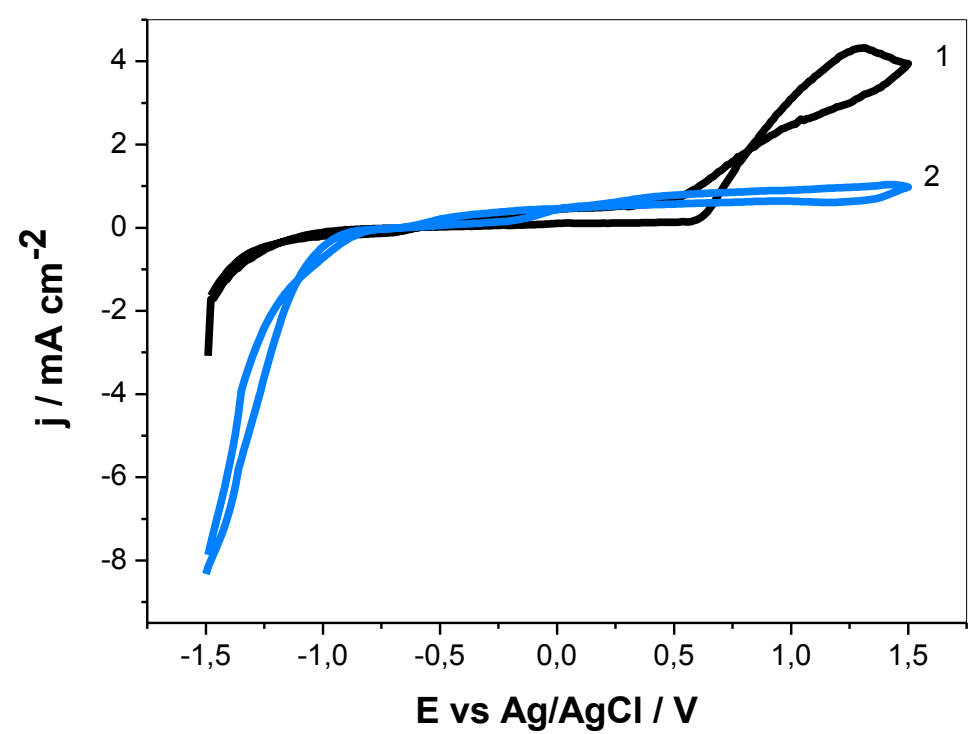

Figura 2: Sobreposição dos voltamogramas cíclicos para: (1) Eletrodeposição de PPy sobre a superfície de alumínio utilizando-se solução aquosa contendo 0,2 mol.L-1 de Py e 0,1 mol.L-1 de DBSA e (2) Influência do DBSA sobre a superfície de alumínio. $v=5 \mathrm{mV}^{-1} \mathrm{~s}^{-1}$.

Conforme apresentado na Curva 1 (Figura 2), o potencial inicial de oxidação do PPy foi de aproximadamente $0,65 \mathrm{~V}$, comportamento similar à eletrodeposição de filmes PPy/DBSA sobre superfícies de aço inoxidável [16]. A ausência de um pico catódico sugere a ocorrência de um processo irreversível de eletropolimerização de Py, que pode estar associada à imobilização conferida pelo ânion volumoso na matriz polimérica durante a dopagem [17], dificultando sua remoção durante o processo de redução. A incorporação de ânions de surfactantes na cadeia polimérica do PPy resulta em filmes com elevada condutividade e excelente propriedade mecânica [18].

A influência do DBSA sobre a superfície do alumínio é apresentada pela Curva 2 (Figura 2), e observam-se menores valores de densidade de corrente para as superfícies de alumínio expostas ao DBSA, na ausência de Py. A presença do ácido orgânico impede a dissolução anódica do metal devido à formação de um filme passivo na interface substrato/eletrólito. Na ausência de Py, micelas são formadas na camada de óxido como resultado da atração hidrofílica entre suas paredes internas e a região hidrofílica do surfactante [19].

A Figura 3 apresenta a sobreposição de voltamogramas para a deposição de filmes de PPy/pTS (Curva 1), e a influência do pTS sobre a superfície de alumínio na ausência de Py (Curva 2); variando-se o potencial de $-1,5 \mathrm{~V}$ a $+1,5 \mathrm{~V}$ a uma velocidade de varredura igual a $5 \mathrm{mV} \cdot \mathrm{s}^{-1}$.

\footnotetext{
* Contribuição técnica ao 69 Congresso Anual da ABM - Internacional e ao 14ํㅡㄹ ENEMET - Encontro Nacional de Estudantes de Engenharia Metalúrgica, de Materiais e de Minas, 21 a 25 de julho de 2014, São Paulo, SP, Brasil.
} 

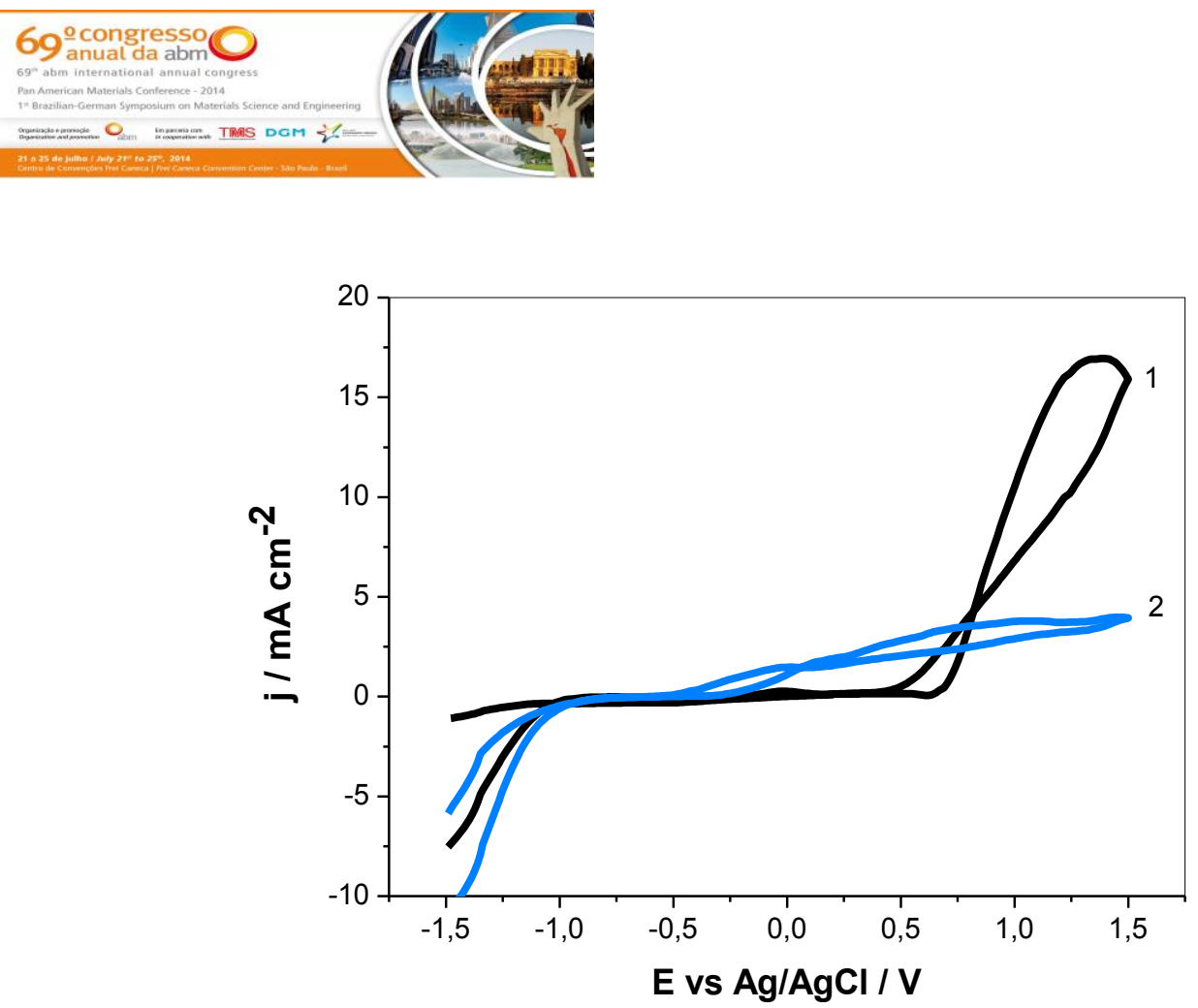

Figura 3: Sobreposição de voltamogramas para: (1) Eletrodeposição de PPy sobre a superfície de alumínio utilizando-se solução aquosa contendo 0,2 mol.L-1 de Py e 0,1 mol.L-1 de pTS e (2) Influência do pTS sobre a superfície de alumínio. $v=5 \mathrm{mV} . \mathrm{s}^{-1}$.

A Curva 1 (Figura 3 ) mostra que o potencial inicial e o pico anódico associado à oxidação de Py são próximos de $0,75 \mathrm{~V}$ e $1,4 \mathrm{~V}$, respectivamente. A ausência de um pico catódico permite inferir a irreversibilidade dos filmes de PPy eletrodepositados em meio contendo o ácido pTS. Estudos prévios desenvolvidos em nosso laboratório mostraram comportamento similar para a deposição de filmes PPy/pTS sobre superfícies de alumínio 99,9\% [20].

Analisando-se a Curva 2 (Figura 3), pode ser observado que o pTS não atua como passivador do substrato de alumínio; comportamento corroborado pelos resultados de Transiente de I x t para a exposição da superfície metálica aos ácidos DBSA e pTS apresentados na Figura 4.

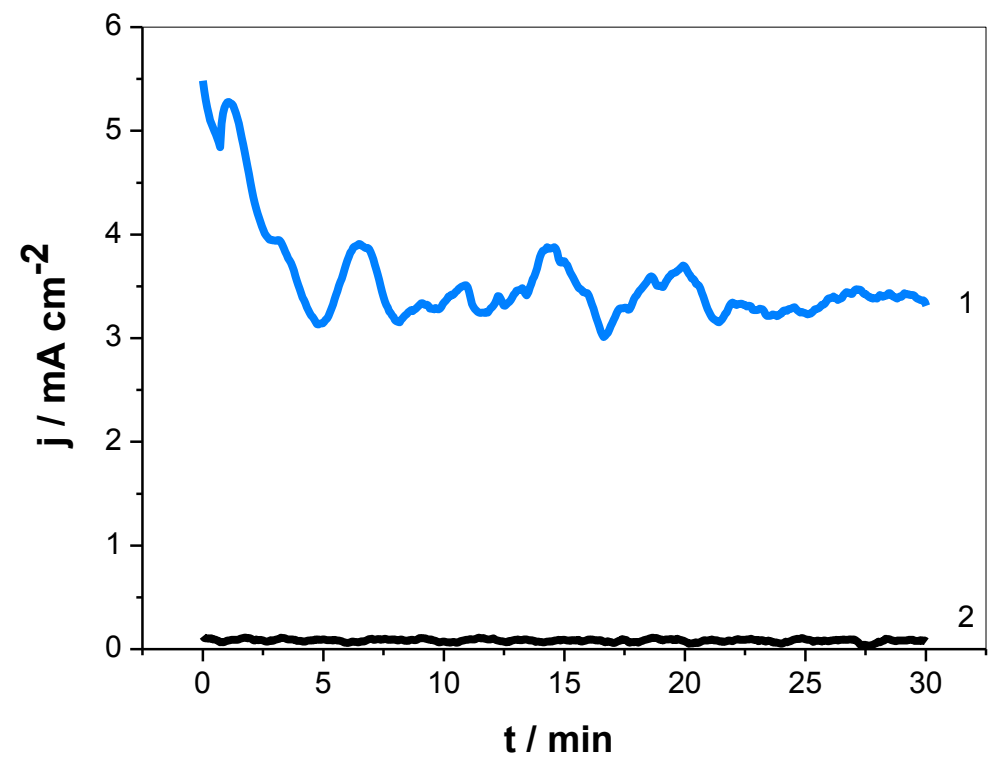

Figura 4: Transiente de corrente versus tempo para a exposição das superfícies de alumínio ao: (1) pTS e (2) DBSA.

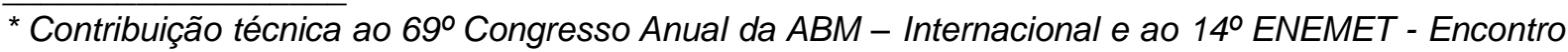
Nacional de Estudantes de Engenharia Metalúrgica, de Materiais e de Minas, 21 a 25 de julho de 2014, São Paulo, SP, Brasil.
} 


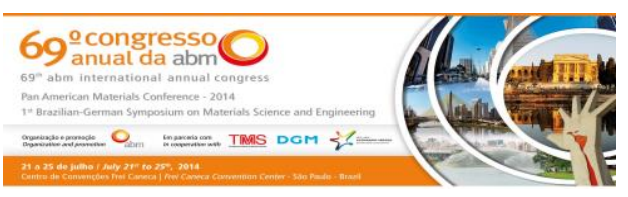

As Curvas de Transiente de $I \times t$ corroboram os resultados apresentados pelos ensaios de voltametria cíclica na ausência de Py. Os maiores valores de densidade de corrente foram observados quando a superfície metálica foi exposta ao meio contendo o ácido pTS (Curva 1).

As possíveis reações químicas são apresentadas nas Equações 1 e 2:

$$
\begin{gathered}
\mathrm{Al}_{(\mathrm{s})} \rightarrow \mathrm{Al}^{3+}{ }_{(\mathrm{aq})}+3 \mathrm{e}^{-} \\
\mathrm{Al}^{3+}{ }_{(\mathrm{aq})}+3 \mathrm{CH}_{3} \mathrm{C}_{6} \mathrm{H}_{4} \mathrm{SO}_{3}^{-}{ }_{(\mathrm{aq})} \rightarrow \mathrm{Al}\left(\mathrm{CH}_{3} \mathrm{C}_{6} \mathrm{H}_{4} \mathrm{SO}_{3}\right)_{3}{ }_{(\mathrm{s})}
\end{gathered}
$$

O Transiente de $I \times t$ (Curva 1) indicou a dissolução do alumínio na etapa inicial (Equação 1), seguida pela queda de corrente associada à formação do complexo $\mathrm{Al}\left(\mathrm{CH}_{3} \mathrm{C}_{6} \mathrm{H}_{4} \mathrm{SO}_{3}\right)_{3}$ (Equação 2) na interface substrato/eletrólito. As oscilações da Curva podem estar associadas à formação de pitting metaestáveis na superfície metálica [21].

A natureza do eletrólito na eletrodeposição de filmes de PPy sobre superfícies de alumínio foram investigadas utilizando-se a técnica potenciostática. As Curvas de $I x$ $t$ para a deposição de PPy em meio aquoso constituído de 0,2 mol. $\mathrm{L}^{-1}$ de Py e 0,1 mol.L-1 de DBSA ou 0,1 mol.L-1 de pTS aplicando-se $1,4 \mathrm{~V}$ durante 900 s são apresentadas na Figura 5.

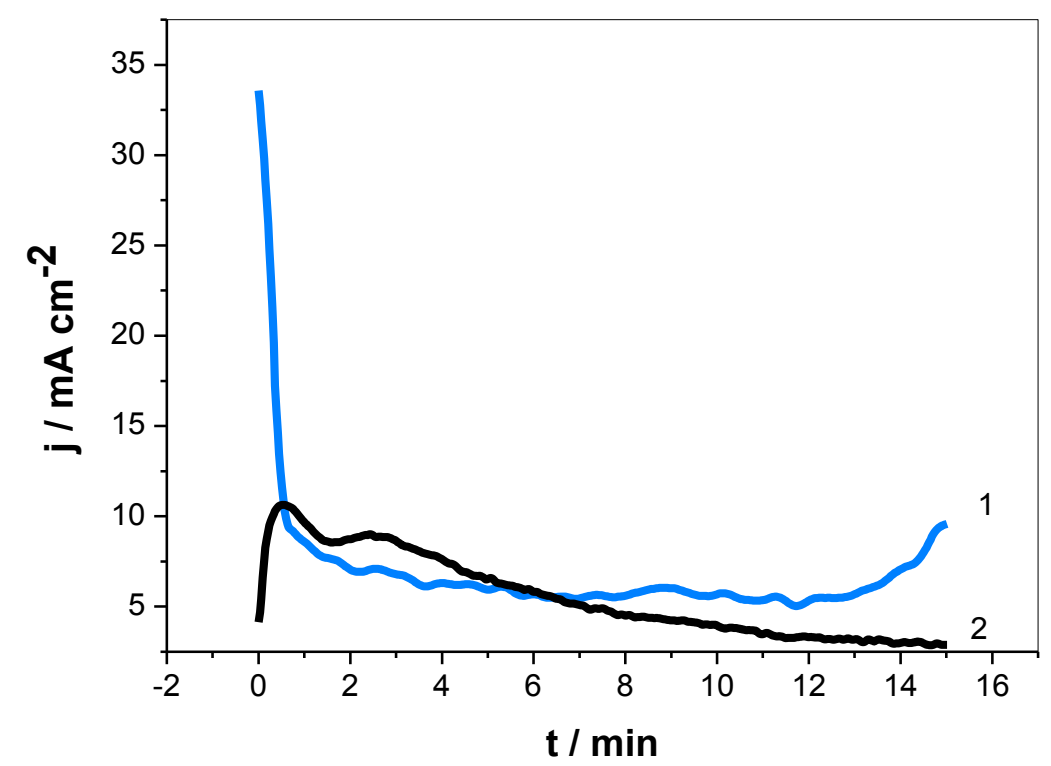

Figura 5: Curvas de corrente versus tempo para a eletrodeposição dos filmes de PPy em solução aquosa contendo 0,2 mol.L $\mathrm{L}^{-1}$ de Py e: (1) 0,1 mol.L-1 de pTS e (2) 0,1 mol.L-1 de DBSA. $v=5$ mV.s ${ }^{-1}$.

A Curva 1 (Figura 5) correspondente à eletrodeposição de filmes de PPy/pTS aplicando-se $1,4 \mathrm{~V}$ indica um brusco crescimento de densidade de corrente associada à formação da camada passiva, seguida pela queda de corrente ligada a nucleação e deposição dos filmes de PPy/pTS sobre o eletrodo de alumínio. A Curva 2 (Figura 5) associada à eletrodeposição de filmes de PPy/DBSA evidencia um súbito decréscimo de corrente em função do tempo, sugerindo a formação de um material com baixa condutividade e menor espessura.

A aplicação de potenciais elevados confere a cadeia polimérica à perda de sua eletroatividade como resultado da incorporação de grupos carbonila no carbono $\beta$ do anel pirrólico, que interrompe as duplas conjugadas e altera a condutividade do

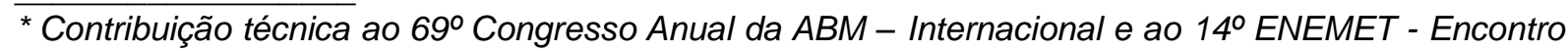
Nacional de Estudantes de Engenharia Metalúrgica, de Materiais e de Minas, 21 a 25 de julho de 2014, São Paulo, SP, Brasil.
} 


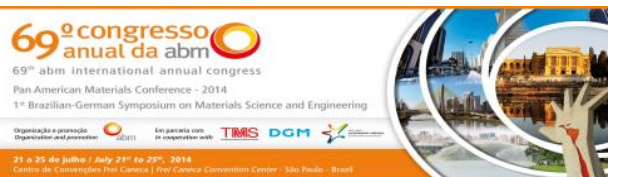

filme [22]. Li e Qian [23] associam a overoxidação do PPy com a oxidação da água; e o mecanismo proposto é apresentado na Figura 6.<smiles>Cc1ccc(-c2ccc(-c3ccc(-c4ccc(C)[nH]4)[nH]3)[nH]2)[nH]1</smiles><smiles></smiles><smiles>Cc1ccc(-c2ccc(-c3ccc(C)[nH]3)[nH]2)[nH]1</smiles>

Figura 6: Mecanismo de overoxidação dos filmes de PPy.

\subsection{Curvas de Polarização Potenciodinâmica e Voltametria Cíclica}

As curvas de polarização obtidas em $\mathrm{NaCl}\left(0,1 \mathrm{~mol}^{-1} \mathrm{~L}^{-1}\right)$ para os filmes de PPy/DBSA e PPy/pTS eletrodepositados em potencial de 1,4V são apresentadas na Figura 7. Os parâmetros de corrosão obtidos a partir das curvas de Tafel são expostos na Tabela 1.

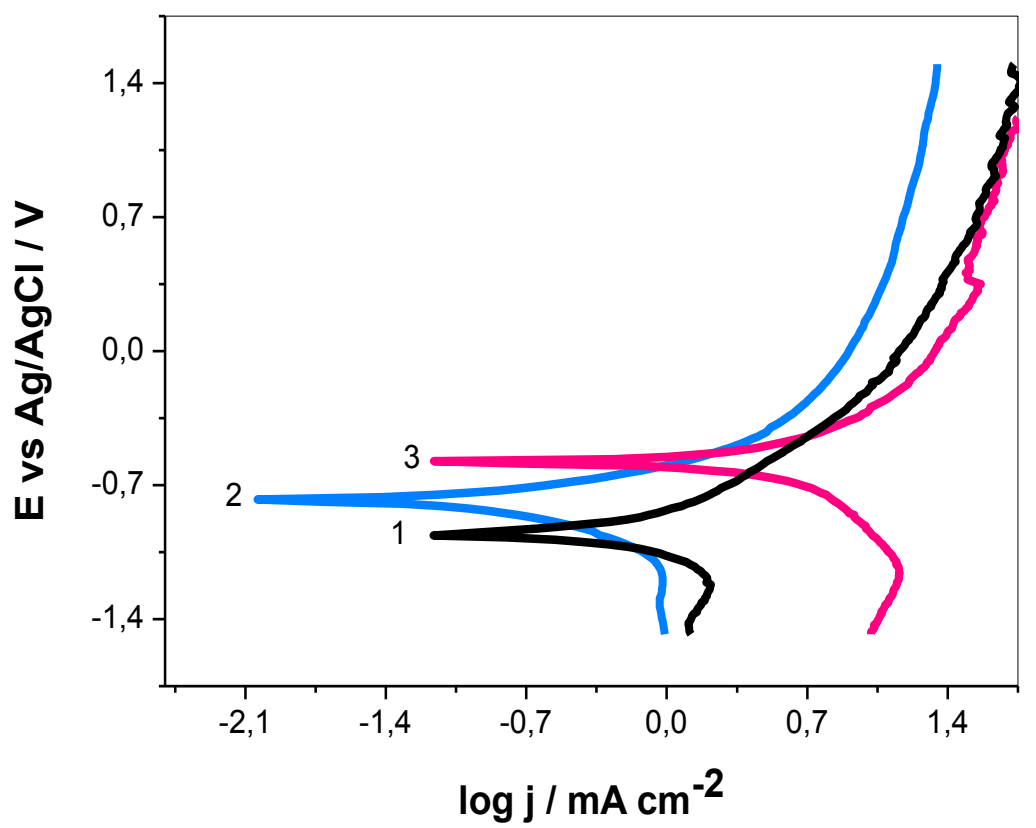

Figura 7: Curvas de polarização obtidas em solução de $\mathrm{NaCl}\left(0,1 \mathrm{~mol}^{\left.-\mathrm{L}^{-1}\right)}\right.$ para as superfícies de alumínio: (1) Apenas polida; (2) Revestidas com filmes de PPy/pTS e (3) Revestidas com filmes de PPy/DBSA. $v=5 \mathrm{mV} \cdot \mathrm{s}^{-1}$.

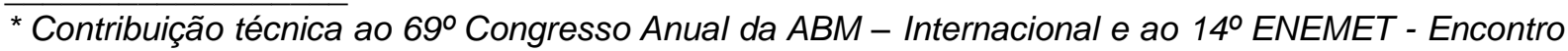
Nacional de Estudantes de Engenharia Metalúrgica, de Materiais e de Minas, 21 a 25 de julho de 2014, São Paulo, SP, Brasil.
} 


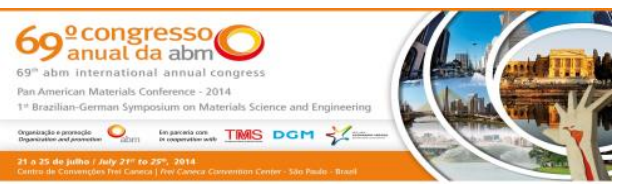

Tabela 1: Parâmetros eletroquímicos para as superfícies de alumínio polarizadas em $\mathrm{NaCl}$.

\begin{tabular}{lcc}
\hline \multicolumn{1}{c}{ Superfícies de Alumínio } & $\mathrm{E}_{\text {corr }}$ Vs. $\mathrm{Ag} / \mathrm{AgCl} / \mathrm{V}$ & $\mathrm{j}_{\text {corr }} / \mathrm{mA} \mathrm{cm}^{-2}$ \\
\hline Apenas Polida & $-0,96$ & 0,65 \\
Recoberta com PPy/DBSA a 1,4V & $-0,92$ & 0,52 \\
Recoberta com PPy/pTS a 1,4V & $-0,77$ & 0,28 \\
\hline
\end{tabular}

Mediante análise das curvas de Tafel e dos parâmetros de corrosão, observa-se o deslocamento do $\mathrm{E}_{\text {corr }}$ para a direção positiva, quando as superfícies de alumínio estão recobertas com filmes de PPy, indicando a presença de um material protetor. Considerando-se que a jcorr está diretamente associada à velocidade de corrosão do metal, pode-se inferir que as superfícies de alumínio recobertas com filmes de PPy/pTS eletrodepositados a 1,4V apresenta melhor desempenho na proteção contra a corrosão, quando comparado aos filmes de PPy/DBSA. As possíveis reações envolvidas durante os ensaios de polarização das superfícies de alumínio são expressas pelas Equações 3, 4, 5 e 6 .

Reações Anódicas:

$$
\begin{aligned}
\mathrm{Al}(\mathrm{s}) & \rightarrow \mathrm{Al}^{3+}(\mathrm{aq})+3 \mathrm{e}^{-} \\
\mathrm{PPy} \text { desdopado } & \rightarrow \mathrm{PPy}_{\text {dopado com } \mathrm{Cl}^{-}+\mathrm{n}^{-}}
\end{aligned}
$$

Reações Catódicas:

$$
\begin{aligned}
& 2 \mathrm{H}_{2} \mathrm{O}(\mathrm{l})+\mathrm{O}_{2}(\mathrm{~g})+4 \mathrm{e}^{-} \rightarrow 4 \mathrm{OH}^{-}(\mathrm{aq}) \\
& \mathrm{PPy} \text { dopado }+\mathrm{n}^{-} \rightarrow \mathrm{PPy} \text { desdopado }
\end{aligned}
$$

Ensaios de voltametria cíclica em $\mathrm{NaCl}\left(0,1 \mathrm{~mol} . \mathrm{L}^{-1}\right)$ para as superfícies de alumínio apenas polida e recobertas com filmes de PPy/DBSA e PPy/pTS eletrodepositados a 1,4V estão apresentados na Figura 8.

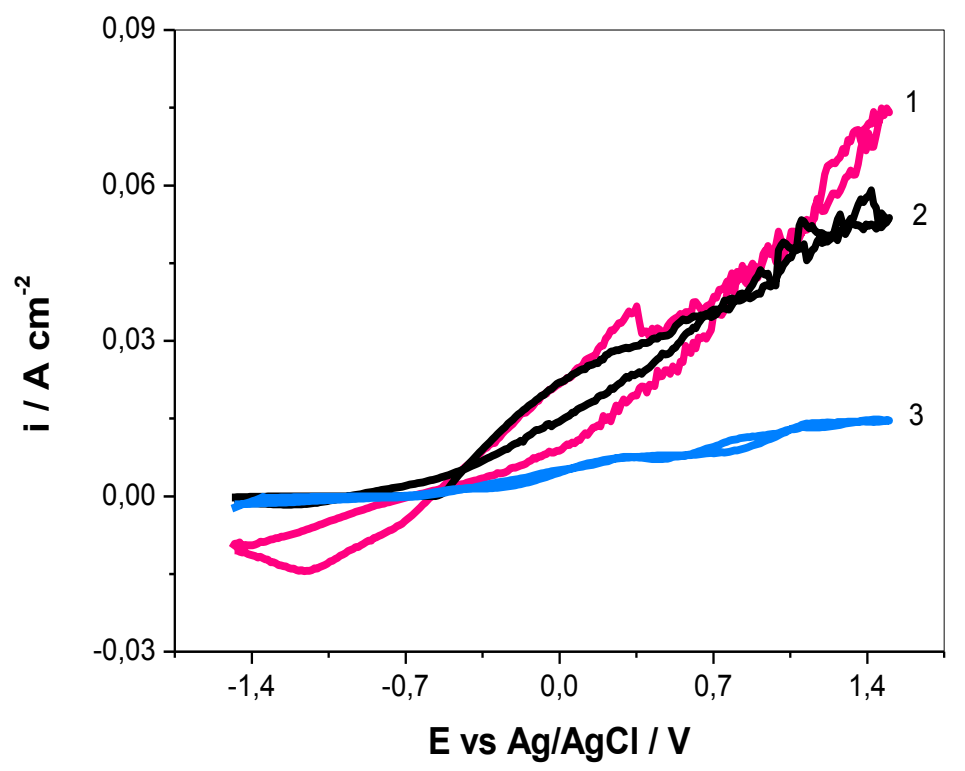

Figura 8: Voltametria cíclica obtida em $\mathrm{NaCl}\left(0,1 \mathrm{~mol} . \mathrm{L}^{-1}\right)$ para as superfícies de alumínio: (1) Revestidas com filmes de PPy/DBSA; (2) Apenas polida e (3) Revestidas com filmes de PPy/pTS. $v=$ $5 \mathrm{mV} . \mathrm{s}^{-1}$.

A Curva 1 (Figura 8) demonstra maiores valores de densidade de corrente para os filmes de PPy/DBSA, quando comparado ao Sistema PPy/pTS (Curva 3).

\footnotetext{
* Contribuição técnica ao 69 Congresso Anual da ABM - Internacional e ao 14ํㅡㄹ ENET - Encontro Nacional de Estudantes de Engenharia Metalúrgica, de Materiais e de Minas, 21 a 25 de julho de 2014, São Paulo, SP, Brasil.
} 


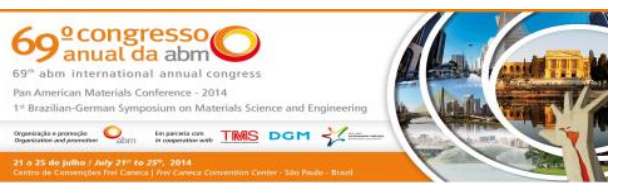

Considerando-se que a adsorção de íons $\mathrm{Cl}^{-}$cresce linearmente com o aumento do potencial [24], pode-se inferir que os filmes de PPy/pTS depositados a 1,4V apresentam comportamento protetor mesmo em elevados potenciais; resultados que confirmam aqueles obtidos com as curvas de Tafel.

\subsection{Caracterização dos Filmes de PPy}

A composição dos filmes de PPy eletrodepositados em meio aquoso contendo o ácido DBSA foi investigada por Infravermelho (FTIR). A Figura 9 apresenta 0 espectro para o Sistema PPy/DBSA, seguida pela Tabela 2 que expõem algumas bandas de absorção indicadas no espectro.

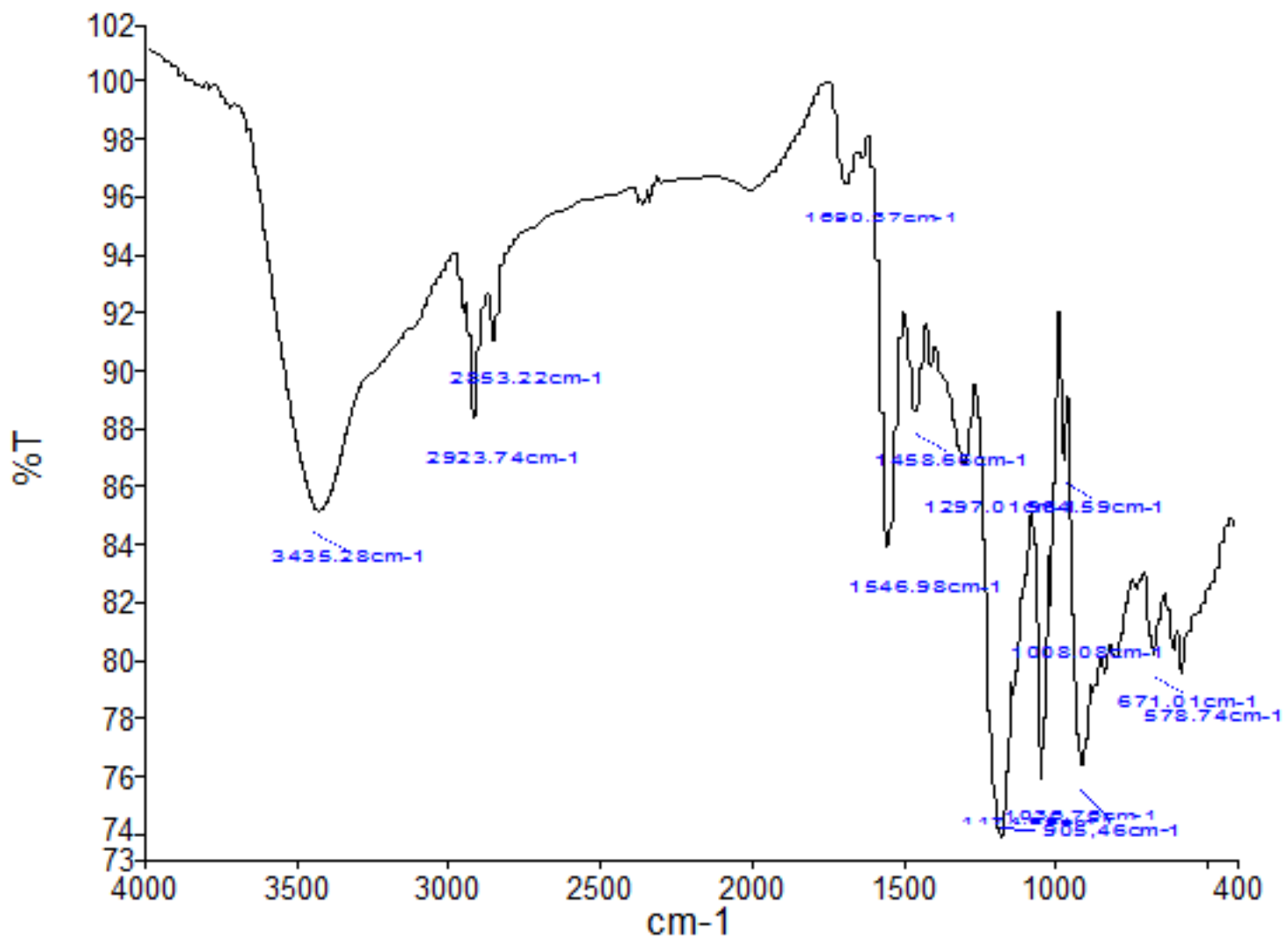

Figura 9: Espectros de FTIR para os filmes de PPy/DBSA.

Tabela 2: Atribuições do espectro de FTIR para os filmes de PPy/DBSA.

\begin{tabular}{cc}
\hline $\mathrm{X}$ & $\mathrm{Y}$ \\
\hline 1690,37 & 96,54 \\
1297,01 & 86,8 \\
1174,66 & 73,82 \\
671,01 & 80,24 \\
\hline
\end{tabular}

A presença de bandas de absorção próximas a $1308 \mathrm{~cm}^{-1}$ (deformação assimétrica axial $\mathrm{O}=\mathrm{S}=\mathrm{O}$ ); $1153 \mathrm{~cm}^{-1}$ (deformação assimétrica axial $\mathrm{O}=\mathrm{S}=0$ ); $671 \mathrm{~cm}^{-1}$ (deformação por estiramento $\mathrm{S}-\mathrm{O}$ ) no espectro do filme de PPy sugerem que o ácido DBSA está incorporado ao filme polimérico. Além disso, a presença da banda em $1690 \mathrm{~cm}^{-1}$ pode estar associada a grupos carbonila (estiramento $\mathrm{C}=\mathrm{O}$ ) formados pela overoxidação da cadeia polimérica.

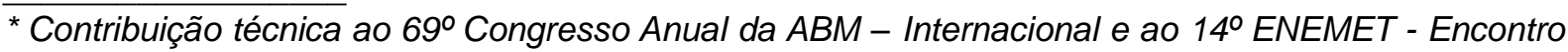
Nacional de Estudantes de Engenharia Metalúrgica, de Materiais e de Minas, 21 a 25 de julho de 2014, São Paulo, SP, Brasil.
} 


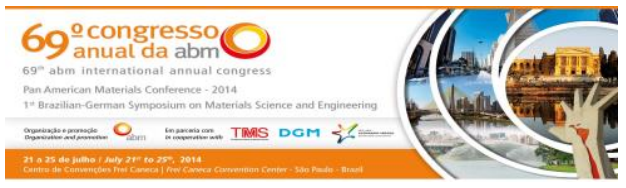

A composição dos filmes de PPy eletrodepositados em meio de ácido pTS é indicada por espectros de FTIR em trabalhos anteriores desenvolvidos em nosso laboratório, ressaltando-se que os espectros para os filmes de PPy/pTS não apresentaram a banda de absorção em $1690 \mathrm{~cm}^{-1}$ associada à overoxidação da cadeia polimérica [20].

A Morfologia dos filmes de PPy eletrodepositados potenciostaticamente em meio contendo os ácidos DBSA e pTS foi investigada por MEV. A Micrografia das superfícies de alumínio recobertas com filmes de PPy/DBSA e PPy/pTS é apresentada na Figura 10.

(a)

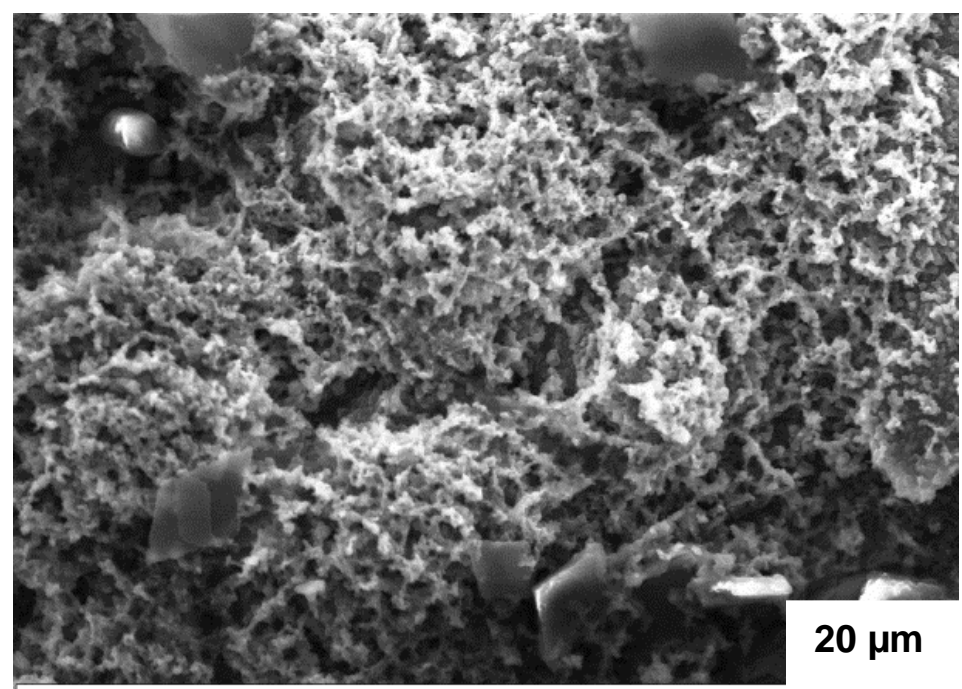

(b)

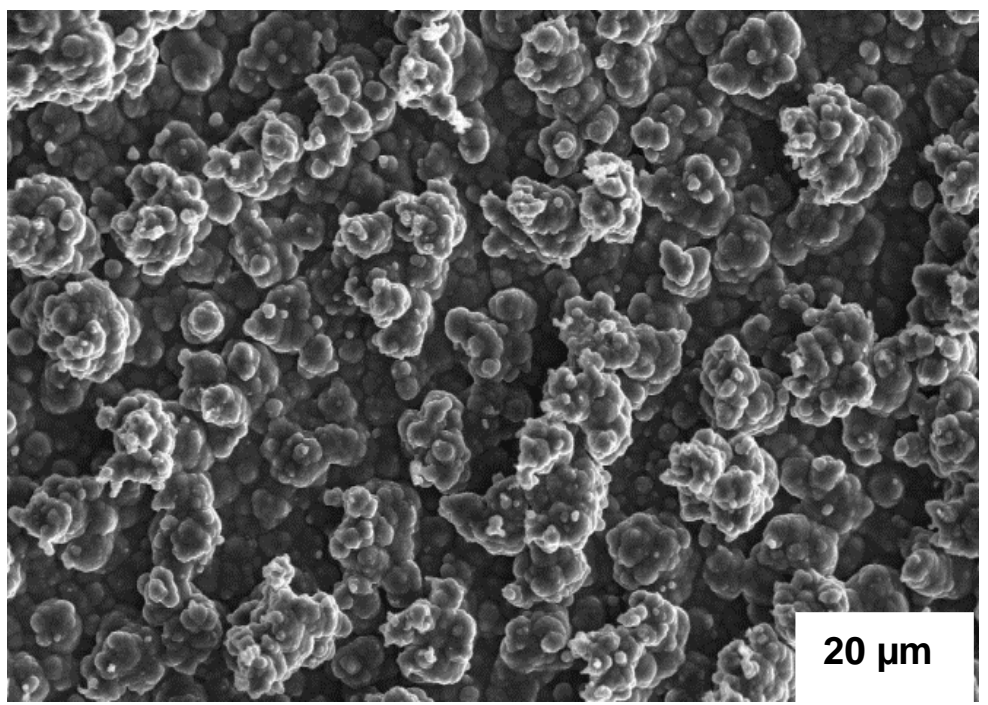

Figura 10: Micrografia (MEV) das superfícies de alumínio revestidas com filmes de: (a) PPy/DBSA formados em 1,4V e (b) PPy/pTS depositados a 1,4V.

A Morfologia dos filmes de PPy/pTS é significativamente mais homogênea do que a obtida para o Sistema PPy/DBSA. A Micrografia dos filmes de PPy/pTS apresenta uma estrutura do tipo cauliflower constituída por grãos micro-esféricos, que tem sido atribuído à dificuldade de intercalação do dopante na cadeia polimérica desordenada [25].

Os filmes de PPy/DBSA apresentaram defeitos estruturais ao longo da cadeia polimérica, associados ao fenômeno irreversível de overoxidação. A degradação do polímero pode resultar em defeitos que permitem a adsorção de espécies agressivas

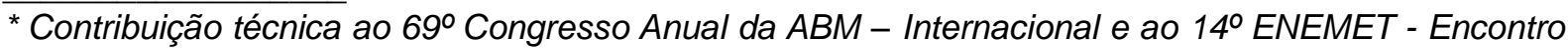
Nacional de Estudantes de Engenharia Metalúrgica, de Materiais e de Minas, 21 a 25 de julho de 2014, São Paulo, SP, Brasil.
} 


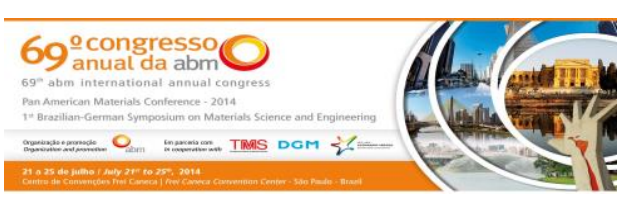

como $\mathrm{Cl}^{-}$durante os ensaios de polarização, podendo justificar os elevados valores de jcorr obtidos com os filmes de PPy/DBSA.

\section{CONCLUSÃO}

A natureza do eletrólito influencia fortemente no processo de eletrodeposição de filmes de PPy. Os resultados de cronoamperometria demonstraram que os filmes de PPy/pTS são mais condutores e espessos, quando comparados aos filmes de PPy/DBSA que sofrem o processo irreversível de overoxidação.

As curvas de Tafel indicaram que os filmes de PPy/pTS são significativamente mais protetores que os filmes de PPy/DBSA. O Sistema PPy/pTS além de resistir à adsorção de íons $\mathrm{Cl}^{-}$em elevados potenciais, apresenta uma Morfologia mais homogênea e uniforme, constituindo-se na melhor condição de proteção da liga de alumínio 2024 contra a corrosão.

\section{Agradecimentos}

Os autores agradecem ao Conselho Nacional de Desenvolvimento Científico e Tecnológico (CNPq) e ao Instituto Federal de Educação, Ciência e Tecnologia de São Paulo (IFSP) pelo financiamento à pesquisa.

\section{REFERÊNCIAS}

1 Bahloul A, Nessark B, Briot E, Groult H, Mauger A, Zaghib K, Julien CM. Polypyrrolecovered $\mathrm{MnO}_{2}$ as electrode material for supercapacitor. Journal of Power Sources, 2013; 240: 267-272.

2 Hashmi SA, Upadhyaya HM. Polypyrrole and poly(3-methyl thiophene)-based solid state redox supercapacitors using ion conducting polymer electrolyte. Solid State Ionics, 2002; 152-153: 883-889.

3 Tsai ML, Chen PJ, Do JS. Preparation and characterization of $\mathrm{PPy} / \mathrm{Al}_{2} \mathrm{O}_{3} / \mathrm{Al}$ used as a solid-state capacitor. Journal of Power Sources, 2004; 133: 302-311.

4 Hughes M, Chen GZ, Shaffer MSP, Fray DJ, Windle AH. Electrochemical capacitance of a nanoporous composite of carbon nanotubes and polypyrrole. Chemistry of Materials, 2002; 14: 1610-1613.

5 Novák P, Muller K, Santhanam KSV, Hass O. Electrochemically active polymers for rechargeable batteries. Chemical Reviews, 1997; 97: 207-281.

6 Weidlich C, Mangold K.-M. Electrochemically switchable polypyrrole coated menbranes. Electrochimica Acta, 2011; 56: 3481-3484.

7 Carquigny S, Segut O, Lakard B, Lallemand F, Fievet P. Effect of electrolyte solvent on the morphology of polypyrrole films: Application to the use of polypyrrole in $\mathrm{pH}$ sensors. Synthetic Metals, 2008; 158: 453-461.

8 Zarras P, Anderson N, Webber C, Irvin DJ, Irvin JA, Guenthner A, et al. Progress in using conductive polymers as corrosion-inhibiting coatings. Radiation Physics and Chemistry, 2003; 68: 387-394.

9 Tallman DE, Spinks G, Dominis A, Wallace GG. Electroactive conducting polymers for corrosion control. Part 1: General introduction and a review of non-ferrous metals. Journal Solid State Electrochemistry, 2002; 6: 73-84.

10 Liu AS, Xavier THA, Cintra EP, Cho LY. Electrodeposition of polypyrrole films on 2024 aluminum alloy in phosphoric acid solution. Materials Science Forum, 2014; 775-776: 225-229.

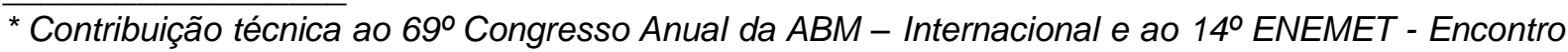
Nacional de Estudantes de Engenharia Metalúrgica, de Materiais e de Minas, 21 a 25 de julho de 2014, São Paulo, SP, Brasil.
} 


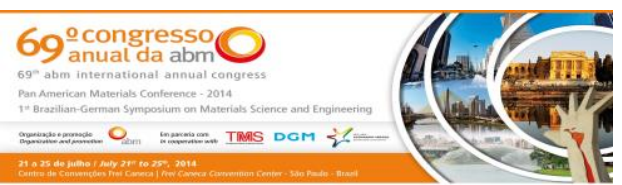

11 Wang LX, Li XG, Yang YL. Preparation, properties and applications of polypyrroles. Reactive e Functional Polymers, 2001; 47: 125-139.

12 Kahalkhali RA. Electrochemical synthesis and characterization of electroactive conducting polypyrrole polymers. Russian Journal of Electrochemistry, 2005; 41: 10711078.

13 Sadki S, Schottland P, Brodie N, Sabouraud G. The mechanisms of pyrrole electropolymerization. Chemistry Society Reviews, 2000; 29: 283-293.

14 Mohammad F. Comparative studies on diffusion behavior of electrochemically prepared polythiophene and polypyrrole: effect of ionic size of dopant. Synthesis Metals, 1999; 99: 149-154.

15 Kaplin DA, Qutubuddin S. Electrochemical synthetized polypyrrole films: effect of polymerization potential and electrolyte type. Polymers, 1995; 36: 1275-1286.

16 Prissanaroon W, Brack N, Pigram PJ, Liesegang J. Co-doped polypyrrole coatings for stainless steel protection. Surface Review and Letters, 2006; 13: 319-327.

17 Ohtsuka T. Corrosion protection of ssteel by conducting polymer coating. Internacional Journal of Corrosion, 2012: 1-7.

18 Warren LF, Anderson DP. Polypyrrole films from aqueous electrolytes: The effect of anions upon order. Journal Electrochemical Society, 1987; 134: 101-105.

19 Naoi K, Takeda M, Kanno H, Sakakura M, Shimada A. Simultaneous electrochemical formation of $\mathrm{Al}_{2} \mathrm{O}_{3}$ /polypyrrole layers (I): effect of electrolyte anion in formation process. Electrochimica Acta, 2000; 45: 3413-3421.

20 Liu AS, Bezerra MC, Cho LC. Electrodeposition of polypyrrole films on aluminum ssurface from a p-toluene sulfonic acid medium. Materials Research, 2009; 12: 503-507.

21 Liu AS, Oliveira MAS. Electrodeposition os polypyrrole films on aluminum from tartrate aqueous solution. Journal of the Brazilian Chemical Society, 2007; 18: 143-152.

22 Rodrigues I, Scharifker BR, Mostany J. In situ FTIR study of redox and overoxidation process in polypyrrole films. Journal Electroanalytical Chemistry, 2000; 491: 117-125.

$23 \mathrm{Li} \mathrm{Y,} \mathrm{Qian} \mathrm{R.} \mathrm{Electrochemical} \mathrm{overoxidation} \mathrm{of} \mathrm{conducting} \mathrm{polypyrrole} \mathrm{nitrate} \mathrm{film} \mathrm{in}$ aqueous solutions. Electrochimica Acta, 2000; 45: 1727-1731.

24 Smialowsha-Szklarska Z. Pitting corrosion of aluminum. Corrosion Science, 1999; 41: 1743-1767.

25 Bazzaoui M, Martins L, Bazzaoui EA, Martins Jl. New single-step electrosynthesis process of homogeneous and strongly adherent polypyrrole films on iron electrodes in aqueous medium. Electrochimica Acta, 2002; 47: 2953-2962. 